\title{
Al in Medicine: Need of Orchestration for High-Performance
}

\author{
Jinwook Choi \\ Department of Biomedical Engineering, Seoul National University College of Medicine, Seoul, Korea
}

Artificial intelligence (AI) has become a buzzword in all areas since AlphaGo beat Lee Sedol in 2016. Autonomous vehicles, access-control facial-recognition systems, and bank withdrawals using facial recognition technology have changed the world substantially. The medical field is no exception. Various AI technologies are already in operation.

The spectrum of medical AI applications is growing rapidly and ranges from reading X-ray images to diagnosing dermatological diseases. In the January 2019 issue of Nature Medicine, Dr. Topol [1] introduced various AI solutions developed for the medical field. He envisioned that they will serve as diagnostic aids for clinicians, improve the workflow in medical institutions, and improve patients' health.

At the Seoul National University Hospital where I work, an AI decision-support system for reading chest posterioranterior (PA) has been in operation since January 2019 [2]. When a doctor selects a patient's chest PA, suspicious lesions detected by AI are displayed in blue in a second window. Although this has not yet been used to predict the patients' disease course, it shows better performance than resident trainees in finding chest nodules.

Watson for Oncology was first installed at the Memorial Sloan Kettering Cancer Center in the United States. It took just a couple of years for seven hospitals in Korea to implement the Watson for Oncology system. Although the appraisal of its effectiveness is not sufficiently beyond expecta-

This is an Open Access article distributed under the terms of the Creative Commons Attribution Non-Commercial License (http://creativecommons.org/licenses/by$\mathrm{nc} / 4.0 /$ ) which permits unrestricted non-commercial use, distribution, and reproduction in any medium, provided the original work is properly cited.

(c) 2019 The Korean Society of Medical Informatics tion, it is certain that Watson's guidelines are very effective in helping decision making and catching up on rapidly growing medical information.

Deep learning research in clinical data processing is also underway to predict the reentry rate of patients. Choi et al. [3] showed that deep learning-based prediction of reentry rate outperformed the conventional machine learning methods.

What is the best way for AI to quickly settle in medicine?

First, it should be recognized that AI is not a complete medical solution. AI cannot solve medical problems completely without human intervention. In particular, as medical care always comes with responsibility, it is necessary to carry out step-wise expert verification at each level. Therefore, it is important to make good use of AI and only adopt it depending on the local conditions.

Second, AI is a targeted technology that has a predefined range of applications. The development of skills should always be clear and targeted, and they should be used in accordance with well-defined goals. It is easy to understand that a Q\&A-based system cannot be used for clinical outcome prediction. This has already been recognized by all developers, but users are not well aware of the fact that it cannot be used for clinical outcome prediction. Scalability of a system should come with meticulous review, especially if the system is highly complicated.

Third, special effort is needed to make good use of AI. Efforts are needed to provide high-quality data. Data from other disciplines are organized in an orderly, homogeneous form, but medical data are often heterogeneous and inconsistent in their spectrum along the time axis. Because of the complexity of medical data, a considerable level of public effort is required to develop high-quality medical AI. 
The Ministry of Health and Welfare in the Republic of Korea started a meaningful task in 2017: creating an education platform for AI. Its goal is to provide quality data through this government project. High-quality X-ray images with annotation are being prepared by the Asan Medical Center, and 3,000 pathology slide images will also be provided through the project. Seoul National University Hospital is also working on a similar project [4].

There is a need for social consent to use patient data. In general, at least 10,000 quality pieces of data should be used to develop AI. This scale of data is usually a result of more than 10 years of data collection at tertiary hospitals. However, patient privacy and consent are stumbling blocks. Medical care is very sensitive and requires careful handling of data. Hence, people will be concerned about the misuse of information or the possibility of personal identification in the future. At this time, it is necessary to provide a guideline that allows the government to fulfill public interest objectives and use data if the probability of misuse is below a reasonable limit.

Finally, it is necessary to develop AI step wise and to use it through proper coordination with experts. AI is like a symphony that cannot be performed with only one instrument. It needs harmony. AI can be the main player in a particular movement, but in some movements, human experts and AI can be equally important players for the symphony. Just as harmony is important for beautiful music, so is true AI for medicine.

\section{ORCID}

Jinwook Choi (http://orcid.org/0000-0002-9424-9944)

\section{References}

1. Topol EJ. High-performance medicine: the convergence of human and artificial intelligence. Nat Med 2019;25(1):44-56.

2. Nam JG, Park S, Hwang EJ, Lee JH, Jin KN, Lim KY, et al. Development and validation of deep learningbased automatic detection algorithm for malignant pulmonary nodules on chest radiographs. Radiology 2019;290(1):218-28.

3. Choi E, Biswal S, Malin B, Duke J, Stewart WF, Sun J. Generating multi-label discrete patient records using generative adversarial networks. Proceedings of the 2nd Machine Learning for Healthcare Conference; 2017 Aug 18-19; Boston, MA. p. 286-305.

4. Pathology AI Platform [Internet]. Sejong, Korea: Ministry of Health and Welfare; c2018 [cited at 2019 Jul 15]. Available from: http://www.wisepaip.org/. 